IdeAs

Idées d'Amériques

15 | 2020

Eau et gestion de l'eau dans les Amériques

\title{
L'eau dans les Amériques
}

Vincent Dubreuil et François Michel Le Tourneau

\section{(2) OpenEdition}

Journals

Édition électronique

URL : http://journals.openedition.org/ideas/7656

DOI : 10.4000/ideas.7656

ISSN : 1950-5701

Éditeur

Institut des Amériques

Référence électronique

Vincent Dubreuil et François Michel Le Tourneau, «L'eau dans les Amériques », IdeAs [En ligne], 15

2020, mis en ligne le 01 mars 2020, consulté le 24 septembre 2020. URL : http://

journals.openedition.org/ideas/7656; DOI : https://doi.org/10.4000/ideas.7656

Ce document a été généré automatiquement le 24 septembre 2020.

\section{(c) $($ i) $(9)$}

IdeAs - Idées d'Amériques est mis à disposition selon les termes de la licence Creative Commons Attribution - Pas d'Utilisation Commerciale - Pas de Modification 4.0 International. 


\title{
L'eau dans les Amériques
}

\author{
Vincent Dubreuil et François Michel Le Tourneau
}

1 La question de l'eau est un enjeu récurrent dans les Amériques. Elle se pose avec une acuité accrue dans le contexte des changements globaux : l'évolution incertaine de la pluviométrie dans un climat qui change, la demande croissante d'une population plus nombreuse et le développement d'activités agricoles ou industrielles extrêmement consommatrices rendent de plus en plus complexe la résolution de l'équation entre l'offre et la demande. Au cours des deux dernières décennies, la multiplication des excès en termes de déficit ou d'abondance a provoqué des "crises de l'eau" successives, justifiant une attention toute particulière portée par les chercheurs des sciences sociales et environnementales.

2 A l'échelle du continent, la ressource en eau est plutôt abondante. Les Amériques représentent par exemple $45,5 \%$ des débits fluviaux cumulés dans le monde, alors qu'elles ne couvrent que $28,5 \%$ des terres émergées. Nombreux sont les fleuves géants, tels le Mississippi, le Saint Laurent ou l'Orénoque, pour ne pas parler de l'Amazone, qui se classe hors catégorie. Le Nord des Amériques, en particulier le Canada, comprend aussi un nombre très important de lacs qui stockent une proportion importante des eaux douces mondiales, et ils sont couverts de manière temporaire ou permanente de neige ou de glace qui représentent elles aussi une part importante de ce stock. Mais cette ressource abondante est marquée par une extrême disparité dans sa répartition, entre les déserts de l'Atacama du Mojave, de nombreuses régions arides comme le Nordeste brésilien ou le Sud-Ouest des États-Unis et, à l'inverse, des régions hyper humides comme l'Amazonie ou les versants Pacifique des Andes colombiennes. Les changements climatiques jouent sur cette répartition, mais plus souvent dans le sens de la multiplication des sécheresses dans les régions déjà peu humides que dans celui d'une extension des régions bien arrosées.

3 L'inégalité de la répartition est aggravée par la «mise en valeur» des ressources hydriques, livrées à une prédation intense au Chili comme dans le Sud-Ouest des ÉtatsUnis (Texas, Arizona, Californie) ou dans une approche plus communautaire dans les oasis andines ou nordestines. Car l'eau est une ressource convoitée qui permet le développement d'activités économiques variées : l'agriculture, irriguée ou non, 
l'industrie et l'énergie, grandes utilisatrices de volumes également, mais aussi les usages industriels et la consommation domestique, ces deux dernières étant le plus souvent urbaines et donc de plus en plus éloignées de la ressource. Pour les approvisionner, des systèmes immenses dérivent les flots de fleuves comme le São Francisco ou le Colorado vers des zones arides situées à des centaines de kilomètres de distance, faisant ainsi de l'eau une marchandise et un élément transporté. Elle est aussi en elle-même un vecteur de transport via les voies fluviales du Saint Laurent ou du Parana-Paraguai. L'eau est ainsi au cœur du monde américain de mobilités croissantes.

4 En Amérique, comme ailleurs dans le monde, l'eau participe aussi à une forme (ou une absence) de lien social aussi bien dans la diversité des pratiques qu'elle permet que dans la représentation que les sociétés se font de cet élément et dans la façon dont elles l'intègrent dans leur développement. En ce sens, l'histoire du rapport à l'eau participe à l'histoire tout court. Depuis la Tennessee Valley Authority, d'innombrables projets ont eu pour objectif une gestion intégrée de l'eau. Des canaux traversant le désert sonorien ou la Caatinga jusqu'aux barrages pharaoniques sur la Columbia, le Xingu ou l'Iguaçu, les logiques d'aménagement à large échelle ont longtemps prévalu. Plus de 12600 barrages de grande portée sont ainsi recensés sur le continent américain ${ }^{1}$, douze des trente plus grands lacs réservoirs et la moitié des dix plus grandes centrales hydroélectriques du monde se trouvent dans les Amériques. Ces projets acquièrent une complexité supplémentaire lorsque les fleuves ou les aquifères concernés traversent les frontières. Or, la dimension continentale des bassins versants implique des négociations ou des rapports de domination entre Etats voisins, ouvrant une fenêtre intéressante sur des enjeux géopolitiques majeurs.

5 Les enjeux, conflits et représentations liés à l'eau se posent aussi à une échelle plus locale et de manière exacerbée dans les métropoles américaines. D'abord via une dégradation dramatique de la ressource à tel point qu'on a pu écrire parfois que des villes tournaient le dos à leur lac ou leur fleuve. On peut ici citer l'extrême pollution de la rivière Tietê, devenue une véritable nuisance dans la ville de São Paulo, mais aussi la transformation de cours d'eau en égouts à ciel ouvert dans de très nombreuses métropoles, y compris en Amérique du Nord comme à Los Angeles. On constate aujourd'hui un mouvement, initié aux Etats-Unis, de réappropriation des berges dans le cadre de politiques municipales volontaristes de "reconquête". Mais dans beaucoup des mégalopoles latines, la situation reste problématique tant $d u$ fait de la pollution des cours d'eau que de l'exposition des populations, souvent les plus modestes, aux risques d'inondations. La diversité des situations américaines quant au rapport à l'eau en ville permet de prendre la mesure des concepts de vulnérabilité et de résilience à l'échelle des territoires.

6 Ce sont ces enjeux globaux et locaux dans leur diversité que souhaite aborder ce numéro thématique de la revue IdeAs sur l'eau dans les Amériques : la dimension environnementale y est fondamentale en mettant l'accent à la fois sur sa dimension physique (quantification des aléas et des ressources) et leur composante sociétale et en prenant explicitement en compte la complexité des jeux des acteurs territoriaux à différentes échelles. En ce sens, les approches géographiques mais aussi historiques, politiques, sociales, économiques et culturelles sont pertinentes pour contribuer à éclairer la thématique couverte par ce volume.

7 Huit articles ont été retenus par le comité éditorial de la revue. Ils couvrent deux pays de l'Amérique du Nord (Etats-Unis, Canada), et une majeure partie de l'Amérique du 
Sud (Brésil, Chili, Argentine, Pérou et Bolivie). Ils représentent également une diversité de milieux: urbains (Buckeye, Curitiba, São Paulo, Nouveau Brunswick), ruraux (Nordeste, Atacama, Arizona), arides, tropicaux ou froids. Les thèmes des conflits et des jeux d'acteurs sont présents dans la plupart des études et concernent des catégories de population très diverses entre les communautés autochtones, les agriculteurs et, bien sûr, les citadins, quels que soient leur niveau et leur cadre de vie : la vulnérabilité de chacun de ces groupes y est clairement posée par les auteurs qui montrent la diversité des situations locales mais aussi la récurrence des problèmes que posent inondations et accès à la ressource en eau.

8 Un premier ensemble de textes aborde la question de l'eau de manière globale au travers de l'étude des politiques, des institutions ou des programmes mis en œuvre pour gérer la ressource et les conflits qui y sont liés. L'article de Florence Laroque (Who Fought for Water and What Did they Fight For ? A Comparative Analysis of Water Conflicts in Latin America between 2000 and 2011) considère l'eau comme un objet à la fois unique et pluridimensionnel de conflit en Argentine, au Chili, en Bolivie et au Pérou: la dimension comparative de ce texte permet d'éclaircir comment et pourquoi les dimensions conflictuelles de l'eau évoluent dans le temps et diffèrent d'un pays à l'autre. Le texte de Carolina Milhorance, Daniela Nogueira et Priscylla Mendes (Do Programa Um Milhão de Cisternas ao Água para Todos: divergências políticas e bricolagem institucional na promoção do acesso à água) aborde le cas du Nordeste brésilien, région aride et semi-aride, où ont été mis en place les programmes "Un million de citernes" puis "Eau pour tous" : les auteurs détaillent les dynamiques d'alliances entre les acteurs sociaux mais aussi les conflits entre les bureaucraties étatiques et les difficultés de la mise en œuvre des politiques publiques au niveau local. Dans un contexte encore plus aride, celui de l'Atacama chilien, Hugo Romero et Manuel Mendez (Dinámica territorial hidrosocial en las geografías altoandinas del norte de Chile: modernización, poder y conflictos en la región de Tarapacá) analysent les processus de production des territoires hydrosociaux dans les hauts plateaux andins de la région de Tarapacá : selon les auteurs, les conflits qui s'y cristallisent autour des communautés traditionnelles sont liés à l'introduction des conceptions occidentales de l'espace dans le cadre du processus de modernisation de la région (irrigation, mines). Le dernier texte, de Franck Poupeau et Joan Cortinas (Le champ des politiques hydriques dans l'Ouest étasunien : éléments d'interprétation des instruments d'action), développe l'étude des politiques hydriques face à la sécheresse dans l'Ouest étasunien: après une cartographie des institutions présentes dans le Colorado River Basin, les auteurs proposent une analyse statistique fine et portent une attention particulière aux instruments de water markets dont les usages varient d'un Etat à l'autre.

9 Le deuxième ensemble de textes concerne plus spécifiquement les espaces urbains où la concentration des hommes et des activités pose les questions de risques, vulnérabilité et conflits d'acteurs de manière exacerbée. Le texte de Guillaume Fortin, Charlotte Poirier, Francis Duhamel et Daniel Germain (Risques d'inondation et vulnérabilité : le cas du bassin versant de la rivière Kennebecasis, Nouveau-Brunswick, Canada) revient sur les concepts utilisés en sciences sociales (aléa, vulnérabilité, risque, perception) et leur importance pour l'adaptation des populations aux changements climatiques: ils montrent comment, à l'échelle du quartier, la réduction du risque inondation passe par une meilleure sensibilisation de la population. Cette question du risque inondation est également le focus de l'article de Gabriela Goudard et Francisco Mendonça (Eventos e episódios pluviais extremos: a configuração de riscos hidrometeorológicos em Curitiba, Paraná - 
Brasil): les auteurs montrent comment cette «ville modèle» du point de vue environnemental est aussi devenue une métropole exposée aux risques liés aux pluies en comparant les éléments physiques (précipitations) et la vulnérabilité des populations, risques éventuellement exacerbés par le manque de coopération intercommunes à l'échelle de l'agglomération. Dans une ville plus grande encore, celle de São Paulo, Neli Aparecida de Mello-Théry, Hervé Théry, Danilo Pereira Sato et Heloisa de Camargo Tozato (Vulnérabilité, risques et conflits liés à l'eau : l'APA de varzea du Rio Tietê, São Paulo, Brésil) détaillent les vulnérabilités et les risques de la plaine inondable de la rivière Tietê via une analyse de l'utilisation des terres: ils montrent comment les pressions de toute sorte (démographiques, immobilières, agricoles et industrielles) mais aussi les enchevêtrements des politiques et projets dans la zone de protection environnementale ont compromis le fonctionnement de la plaine alluviale. Finalement, l'article d'Anne-Lise Boyer et Rebecca F.A. Bernat (De la luzerne aux masterplanned communities : enjeux de la gestion de l'eau sur un front d'urbanisation, le cas de Buckeye en Arizona) expose les modalités de la gestion de l'eau à l'échelle municipale étasunienne et en montre les contradictions : le laisser-faire des divers échelons de la puissance publique (État, municipalités) concernant le foncier limite l'efficacité des dispositifs mis en place pour réguler l'exploitation et la pression sur la ressource en eau.

\section{NOTES}

1. Commission internationale des grands barrages (www.icold-cigb.org).

\section{AUTEURS}

\section{VINCENT DUBREUIL}

Vincent Dubreuil est Professeur à l'Université Rennes 2 et membre de l'UMR 6554 LETG (Littoral Environnement Télédétection Géomatique). Géographe climatologue, ses travaux portent sur les risques (sécheresse) et les changements climatiques en France et au Brésil de l'échelle locale (îlots de chaleur urbain) à l'échelle régionale (impact de la déforestation en Amazonie sur le climat) et utilisent de manière privilégiée les données de satellites. Il coordonne ou participe à plusieurs projets de recherches en collaboration avec le Brésil (CNRS, ANR, COFECUB, H2020, FAPESP...) et a été professeur invité dans les universités de Brasilia, Presidente Prudente, Londrina et Fortaleza.

\section{FRANÇOIS MICHEL LE TOURNEAU}

François-Michel Le Tourneau est directeur de recherche au CNRS et directeur-adhoint de l'UMI iGLOBES (CNRS-ENS-Université d'Arizona). 